\title{
Avaliação do conhecimento sobre Iniciação Científica em alunos de Medicina
}

\author{
Assessment of Scientific Initiation Knowledge in Medical Students
}

João Pedro de Resende Côrtes, Sara Cristine Marques dos Santos, Thaís Lemos de Souza Macedo, João Carlos de Souza Côrtes Junior, Paula Pitta de Resende Côrtes, Ivana Picone Borges de Aragão.

Como citar esse artigo. Côrtes, J.P.R;

dos Santos, S.C.M; Macedo, T.L.S;

Junior, J.C.S.C; Côrtes, P.P.R; Aragão,

I.P.B. Avaliação Do Conhecimento

Sobre Iniciação Científica Em

Alunos De Medicina. Revista de

Saúde. 2021 Abr./Jul.; 12 (2): 02-07.

\section{Resumo}

A participação do aluno em atividades de iniciação cientifica (IC)proporciona a sua inserção precoce na área de pesquisa, com consequente aquisição de conhecimentos, que serão importantes para sua vida profissional, além servir de estímulo para o seu futuro ingresso, em programas de pós-graduação. O objetivo do presente estudo foi analisar o panorama do conhecimento sobre IC por estudantes de medicina. Foi realizado um estudo observacional e transversal sobre o conhecimento científico em alunos de medicina, através da aplicação de questionário anônimo online, sobre temas relacionados à IC, como: currículo Lattes; Plataforma Brasil; produção científica.O total de 224 entrevistados, 176 (78,57\%) não atuam em iniciação científica, $181(80,8 \%)$ desconhecem os trâmites para obtenção de um parecer pelo Comitê de Ética em Pesquisa, 103 (45,98\%) nunca produziram um tema-livre e não sabem o que é e 123 (54,91\%) nunca produziram um artigo científico. Mais da metade dos alunos, 117 (52,23\%), não possuíam o cadastro no currículo Lattes. Três a cada quatro estudantes de medicina não atuam na iniciação científica e, a grande maioria desconhece os trâmites para à produção de pesquisa científica e metade nunca produziu trabalho de tema livre ou artigo.

Palavras-chave: Avaliação da Pesquisa em Saúde; Avaliação de Programas e Instrumentos de Pesquisa; Conhecimento; Estudantes de Medicina.

\begin{abstract}
The participation of students in scientific initiation (CI) activities provides their early insertion in the research area, with consequent acquisition of knowledge, which will be important for their professional life, besides serving as a stimulus for their future entry into graduate programs. The objective of this study was to analyze the panorama of knowledge about CI among medical students. Observational and cross-sectional study on scientific knowledge in medical students, by applying an anonymous online questionnaire on topics related to HF, such as: Currículo Lattes; Plataforma Brasil; scientific production. Of 224 interviewers, 176(78.57\%) did not work in scientific initiation, 181(80.8\%) did not know how to obtain an opinion from the Research EthicsCommittee, 103(45.98\%) had never produced a free subject and did not know what it is and 123(54.91\%) had never produced a scientific article. More than half of the students, $117(52.23 \%)$, did not have the register in the Currículo Lattes. Three out of four medical students do not act in the scientific initiation and, the great majority does not know the procedures for the production of scientific research and half never produced a free theme work or article.

Keywords: Health Research Evaluation; Evaluation of Research Programs and Tools; Students, Medical.
\end{abstract}

\section{Introdução}

A iniciação cientifica (IC) na graduação é responsável pela introdução do aluno na área da pesquisa, possibilitando que ele assuma, junto com seu orientador, a posição de pesquisador, tornando-se capaz de adquirir conhecimentos teóricos e metodológicos no processo de desenvolvimento do projeto de pesquisa $(\mathrm{PP})^{1}$. Essa inserção costuma ser gradual, gerando ao acadêmico,crescimento na área científica, estimulando a criação de perguntas e a busca por respostas, trilhando o caminho para futura inserção em programas de pós-graduação,juntamente ao enriquecimento de seu currículo².

O estudante, ao definir seu tema de estudo, deverá ser capaz de analisar, interpretar e propor ideias de forma coerente a respeito do assunto, podendo discutir criticamente o conteúdo, além da aquisição da capacidade de sintetizar os achados em suas argumentações ${ }^{3}$.

Afiliação dos autores: ' Discente do Curso de Medicina da Universidade de Vassouras, Rio de Janeiro, Brasil. ORCID: https://orcid.org/0000-0002-5873-3114

${ }^{2}$ Discente do Curso de Medicina da Universidade de Vassouras, Rio de Janeiro, Brasil. ORCID: https://orcid.org/0000-0002-8205-8112

${ }^{3}$ Discente do Curso de Medicina da Universidade de Vassouras, Rio de Janeiro, Brasil. ORCID: https://orcid.org/0000-0002-7667-6061

${ }^{4}$ Doutor em medicina, docente e Pró-Reitor de Ciências Médicas da Universidade de Vassouras, Vassouras, Rio de Janeiro, Brasil. ORCID: https://orcid.org/0000-0002-3263-5129

${ }^{5}$ Mestre em medicina, docente do Curso de Medicina e coordenadora do Curso de Medicina da Universidade de Vassouras, Vassouras, Rio de Janeiro, Brasil. ORCID: https://orcid.org/0000-00015935-7045

${ }^{6}$ Doutora em medicina e docente do Curso de Medicina e do Mestrado de Ciências Médicas da Universidade de Vassouras, Vassouras, Rio de Janeiro, Brasil. ORCID: https://orcid.org/0000-00024295-0165

*Email de correspondência: saracmarques@icloud.com

Recebido em: 27/11/20. Aceito em: 04/06/21. 
A IC na medicina proporciona a formação de um profissional diferenciado, onde a experiência com o orientador, que é uma figura com grande poder de influência, irá ser responsável por passar ao aluno, qualidades e valores morais que serão empregados em sua prática clínica futuramente 4 .

O desenvolvimento científico está correlacionado com o desenvolvimento de um país, tornando as informações mais acessíveis a toda população, promovendo inovações nas ciências e tecnologias 5 .Apesar de, mundialmente, estar ocorrendo um aumento do interesse pela área científica na formação médica $^{6}$, no Brasil, a IC ainda não é distribuída de forma igualitária entre todos os estudantes e, de acordo com o Enade 2007, apenas 39\% declararam possuir a disciplina de IC, de forma regulamentar no seu curso ${ }^{1}$.

Um desafio enfrentado por aqueles que querem seguir esse caminho da pesquisa científica éa ausência de incentivo e auxílio financeiro, por parte das instituições. Os cortes orçamentários nos fundos direcionados para as pesquisas são cada vez mais frequentes, dificultando a manutenção de laboratórios, aquisição de materiais e oferta de bolsas de iniciação cientifica e pós graduação ${ }^{7}$.

Outro fator dificultador encontrado pelos estudantes é a falta de condições favoráveis e oportunidades, dentro do ambiente da faculdade. Tornando assim, aqueles que conseguem estar envolvidos em atividades de pesquisa, alunos diferenciados, nos quais alguns pesquisadores brasileiros procuram investigar suas qualidades e perfis ${ }^{8}$.

Espera-se que o graduando da atualidade seja ativo na construção de seu conhecimento, sabendo questionar e assumindo posto de destaque em sua formação e é nesse ponto que a pesquisa entrará, como fator fundamental no processo educativo9 .

Algumas instituições de ensino já adotaram a indissociabilidade dos pilares acadêmicos, que consistem em ensino, pesquisa e extensão ${ }^{9}$, incentivando a produção científica, ainda durante a graduação, e desenvolvendo pensamento crítico e fundamentação para leitura de artigos importantes, para a formação médica assistencial, atuando como um método de educação continuada ${ }^{6,10}$.

O objetivo do presente estudo foi analisar o conhecimento do estudante de medicina a respeito da IC, de modo a identificar suas possíveis dificuldades e dúvidas sobre a produção científica,visando poder atuar nas lacunas encontradas, através de capacitações, posteriormente.

\section{Metodologia}

Estudo observacional, descritivo, quantitativo e transversal sobre o conhecimento em relação às atividades de iniciação científica, sob o número de parecer 4.176.241 no Conselho de Ética em Pesquisa (CEP).

Cálculo amostral de, no mínimo 201 alunos, realizado através do site comentto ${ }^{11}$, baseado em 1.092 alunos matriculados no curso de medicina, com erro calculado de 5\%, nível de confiança de $95 \%$ e distribuição da população homogeneamente (80:20).

Realizado através da aplicação de questionário anônimo online, original e elaborado pela autoria, com fins de avaliar a população estudada acerca de seu conhecimento em iniciação científica.

Critérios de inclusão na pesquisa era ser aluno do curso de medicinada universidade com idade superior a 18 anos e de exclusão ser de outros cursos ou instituições e possuir idade inferior a 18 anos.

O questionário foi confeccionado pelos autores do estudo, contendo 21 questões que abordavam a idade; período da graduação em andamento; gênero, conhecimento sobre as plataformas científicas Lattes (PL) e Plataforma Brasil (PB); produção de tema livre e artigos científicos; sobre projetos de pesquisa e extensão e atuação na área científica.

Os dados obtidos foram tabulados e analisados qualitativamente, através do programa Excel, assim comopara a análise estatística descritiva.

O total de alunos foi dividido em dois grupos, quanto ao ciclo do curso de medicina: grupo dociclo básico e do ciclo clínico,para análise comparativa.

\section{Resultados}

Participaram do estudo, 224 estudantes, sendo que $125(55,80 \%)$ delesse encontravam no ciclo básico do curso e $99(44,20 \%)$ no clínico.

Os estudantes foram avaliadosem relação à experiência com a Plataforma Lattes, sendo evidenciado que $151(67,41 \%)$ informaram saber o que era a PL, $45(20,09 \%)$ desconheciam e $28(12,50 \%)$, sabiam parcialmente. Mais da metade, 117(52,23\%) alunos, não possuíam o cadastro no currículo Lattes (CL) e 107 $(47,70 \%)$ o possuíam (Tabela 1 e Figura 1$)$.

Ao serem questionados sobre a atualização do CL, a maioria do grupo total de 224 alunos, informou desconhecer como fazer a inserção de suas atividades no currículo Lattes, com $160(71,42 \%)$ respostas, contra apenas $64(28,57 \%)$ que a conheciam.

Entre 141 alunos que responderam o questionamento sobre o costume demanter a atualização do CL, a maioria com 107 (75,89\%) acadêmicos o negaram, enquanto $34(24,11 \%)$ informaram que o atualizava.

No que tange a Plataforma Brasil (PB), os acadêmicos foram interrogados sobre o conhecimento da existência da PB, havendo $128(57,14 \%)$ respostas de 
Tabela 1. Respostas do questionário de acordo com o ciclo cursado. Números absolutos e proporção.

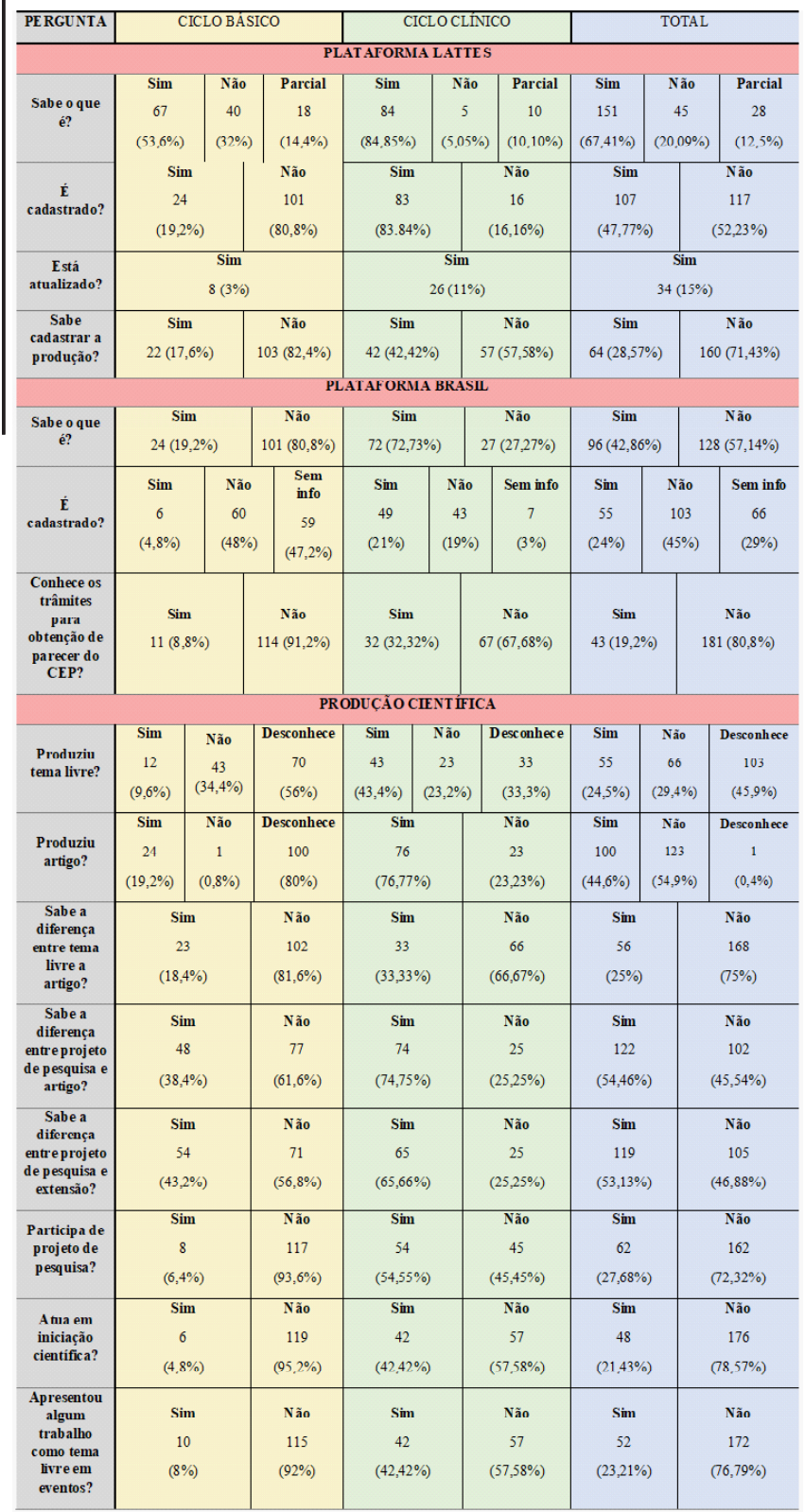

\section{VOCÊ POSSUI CURRÍCULO LATTES?}

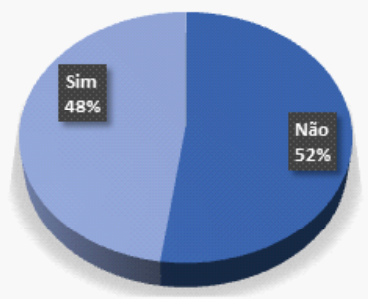

Figura 1. Estudantes que possuem cadastro no Currículo Lattes.

desconhecimento enquanto $96(42,86 \%)$ a conheciam. Quando foram inquiridos sobre serem cadastrados na $\mathrm{PB}$, houve um total de 158 respostas, entre elas, haviam $103(65,19 \%)$ negativas e $55(34,81 \%)$ afirmativas (Figura 2).

\section{VOCÊ CONHECE A PLATAFORMA BRASIL?}

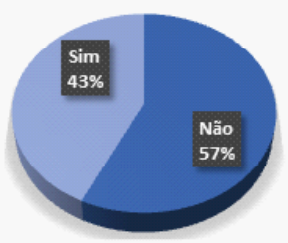

Figura 2. Conhecimento sobre a Plataforma Brasil.

Ao serem questionados se conheciam os trâmites para obtenção de parecer pelo Conselho de Ética em Pesquisa, 181 (80,80\%) desconheciam, contra $43(19,20 \%)$ que o conheciam.

Quanto a produção de temas livres (TL), 103 $(45,98 \%)$ declararam não saberem do que se tratava, 66 $(29,46 \%)$ já ouviram falar, mas nunca o produziram e $55(24,55 \%)$ já o produziram.

No questionamento sobre terem produzido um artigo científico (AC), $123(54,91 \%)$ alunos informaram que nunca o fizeram, $100(44,64 \%)$ já o produziram e $1(0,45 \%)$ desconhecia (Figura 3). Porém, quando perguntados se conheciam a diferença entre um TL e um AC, 168 (75\%) estudantes desconheciam a diferença,

\section{VOCÊ JÁ PRODUZIU UM ARTIGO CIENTÍFICO?}

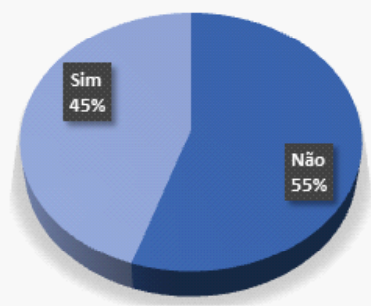

Figura 3. Produção de artigo pelos estudantes.

contra $56(25 \%)$ que a conheciam.

Em relação à diferença entre $\mathrm{PP}$ e $\mathrm{AC}, 102$ $(45,54 \%)$ estudantes não tinham conhecimento e 122 $(54,46 \%)$ o tinham. Entre PP e de projeto de extensão (PE), 105 (46,88\%) desconheciam e 119 (53,13\%) o conheciam.

Questionados se faziam parte de algum PP, 162 $(72,32 \%)$ não participavam e $62(27,68 \%)$ sim, bem como sobre a atuação acadêmica como aluno de IC, sendo obtidas $176(78,57 \%)$ respostas negativas e 48 $(21,43 \%)$ positivas (Figura 4).

Quanto a apresentação de trabalhos científicos, tipo TL, em eventos científicos, 172 (76,79\%) nunca os apresentaram e $52(23,21 \%) \mathrm{sim}$. Entre aqueles que tiveram a experiencia de ter apresentado TL em evento científico, 28 (53,85\%) alunos já haviam apresentado 


\section{VOCÊ ATUA EM INICIAÇÃO CIENTÍFICA?}

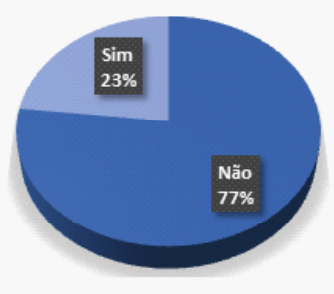

Figura 4. Conhecimento sobre a Plataforma Brasil.

entre 1 e $3,8(15,38 \%)$ entre 4 e 6 e $3(5,77 \%)$ entre 7 e 9 e $13(25 \%) 10$ ou mais trabalhos,

O grupo total foi abordado sobre quais atividades os estudantes já haviam participado, em relação à pesquisa científica, sendo identificadas 44 (19,64\%) respostas informando ter sido através da participação na elaboração de um PP, 18 (8,04\%) haviam preenchido os dados na Plataforma Brasil, 21 (9,38\%) submeteram PP à coordenadoria de pesquisa, $32(14,29 \%)$ atuaram na coleta de dados do PP, 19 (8,48\%) da inserção de dados nas planilhas, $19(8,48 \%)$ da interpretação de dados e análise estatística, 38 (16,96\%)elaboraram TL/resumos e $28(12,5 \%)$ produziram artigos junto ao grupo de pesquisa (Figura 5).

\section{PARTICIPAÇÃO EM ATIVIDADES DE PESQUISA}

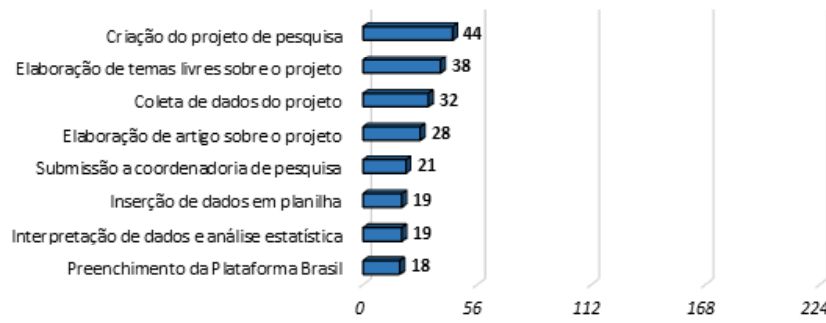

Figura 5. A participação dos estudantes em atividades de pesquisa.

Houve a participação de estudantes do $1^{\circ}$ ao $12^{\circ}$ período, onde a média foi aproximadamente o $5^{\circ}$ (4,758928571), com desvio padrão de 3,476366166, variância da amostra de 12,08512172 , erro padrão de 0,232274485 , curtose $-1,292300651$, assimetria de 0,466789808 e nível de confiança (95\%) de 0,457733795 .

\section{Ciclo básico}

Ao estratificar os resultados por ciclo do curso, no básico, dos 125 alunos, 67 (53,6\%) sabiam o que era a PL, 40 (32\%) desconheciam e $18(14,40 \%)$ conheciam parcialmente, sendo que 101 (80,8\%) não eram cadastrados e $24(19,20 \%)$ sim. Neste grupo, 103 $(82,40 \%)$ deles não sabiam como cadastrar sua produção científica e certificado de comparecimento em eventos, contra $22(17,60 \%)$ que sabiam fazê-lo (tabela 1).

Sobre o conhecimento a respeito da $\mathrm{PB}$, $101(80,80 \%)$ declararam desconhecimento e 24 $(19,20 \%)$ a conheciam. Ao serem questionados sobre o conhecimento dos trâmites de obtenção de aprovação de PP pelo CEP, 114 (91,20\%) desconheciam e 11 (8,80\%) informaram conhecer.

Quanto a produção científica, 70 (56\%) não sabiam o que é um TL, 43 (34,40\%) nunca produziram e $12(9,6 \%)$ sim. Em relação à produção de $\mathrm{AC}, 100$ $(80 \%)$ nunca haviam produzido, $24(19,20 \%)$ sim e 1 $(0,80 \%)$ não sabia do que se tratava.

Sobre a pergunta relacionada à diferença entre PP e AC, 77 (61,60\%) desconheciam e 48 (38,40\%) sabiam; a diferença entre PP e PE era desconhecida por $71(56,80 \%)$ e conhecida por $54(43,20 \%)$; e a diferença entre TL e AC foi declarada desconhecida por $102(81,60 \%)$ e conhecida por $23(18,40 \%)$. Do total, $115(92 \%)$ nunca apresentaram trabalhos em eventos e $10(8 \%)$ já haviam apresentado.

Quando questionados se atuavam como IC na universidade, $119(95,20 \%)$ não participavam e 6 $(4,80 \%) \operatorname{sim}$.

\section{Ciclo clínico}

Quanto ao ciclo clínico, dos 99 que preencheram o questionário, 84 (84,85\%) conheciam a PL, 10 (10,1\%) parcialmente e $5(5,05 \%)$ não conheciam. Um total de $83(83,84 \%)$ possuía cadastro na PL e $16(16,16 \%)$ não; $57(57,58 \%)$ deles informaram não saber como cadastrar sua produção no CL e 42 (42,42\%) sabiam (tabela 1).

Em relação ao questionamento sobre a $\mathrm{PB}, 72$ (72,73\%) já conheciam e 27 (27,27\%) a desconheciam. Entre o total de 99 estudantes do ciclo clínico, um grupo de 92 participantes responderam em relação ao cadastro na $\mathrm{PB}$, onde $43(46,74 \%$ ) não possuem cadastro e $49(53,26 \%)$ possuem, enquanto $67(67,68 \%)$ desconhecem os trâmites para obtenção de parecer pelo CEP e 32 (32,32\%) o conhecem.

Na seção de produção científica, 43 (43,43\%) já produziram um TL, $33(33,33 \%)$ não sabem o que é um TL e $23(23,23 \%)$ nunca o fizeram; da produção de $\mathrm{AC}, 76(76,77 \%)$ já escreveram e 23 (23,23\%) não; $66(66,67 \%)$ não sabem a diferença entre TL e AC e 33 $(33,33 \%)$ sabem; em relação a diferença de PP e AC, 74 (74,75\%) sabem dizer e $25(25,25 \%)$ não; entre PP e PE, 65 (65,66\%) sabem diferenciar e 34 (34,34\%) não sabem. Informaram nunca terem apresentado trabalho em eventos científicos 57 (57,58\%), onde 42 (42,42\%) informaram sim. Na atuação em iniciação científica, 57 $(57,58 \%)$ não atuam e $42(42,42 \%)$ sim. 


\section{Discussão}

Cerca de metade dos alunos não possuem cadastro na plataforma Lattes, onde cerca de $70 \%$ não sabe como atualizá-la, fazendo com que grande parte dos que o possuem, mantenham desatualizado.

É sabido que o currículo Lattes é de grande importância durante a vida acadêmica, sendo uma forma de padronização da inserção e difusão das atividades científicas de alunos e professores, utilizado pela maioria das instituições de fomento à pesquisa ${ }^{12}$. Ele abrange o registro da produção científica nacional, sendo pré-requisito para aqueles que irão seguir o caminho da pesquisa, pois podem ser usados na seleção de candidatos a bolsas e financiamento de projetos ${ }^{13}$.

$\mathrm{O}$ desconhecimento e a dificuldade de preenchimento das informações no currículo Lattes faz com que haja uma divulgação precária das pesquisas em andamento no país, assim como poderá ser um fator prejudicial a esses alunos, tanto no mercado de trabalho e processos seletivos de residência médica, quanto na sua pós-graduação acadêmica.

A PB ainda é majoritariamente desconhecida pelos alunos, assim como os trâmites para obter parecer do CEP para realização de uma pesquisa. Ela é responsável pela criação e alimentação de uma base a nível nacional de pesquisa, sendo responsável pelo encaminhamento ao CEP mais próximo, permitindo um acompanhamento evolutivo dos projetos, através do envio de relatórios ${ }^{14}$.

Para a submissão de projetos em seu sistema, é necessário que os autores sejam cadastrados. O queevidencia a necessidade de uma maior difusão de conhecimento a seu respeito.

Entre as atividades de pesquisa, o maior percentual de participação foi durante a elaboração de projeto, porém, contou com menos de $20 \%$ do total de estudantes.

A produção de trabalhos científicos é pouco frequente, sendo observada em menos da metade do total de estudantes entrevistados.

Acredita-se que um incentivador para a produção científica seja a participação em ligas acadêmicas, pois através do contato mais aprofundado com temas da área de interesse dos estudantes e o auxílio dos professores responsáveis, haverá um maior estímulo para a escrita de trabalhos, predominantemente, relatos de experiência ou editoriais ${ }^{15}$.

Grande parte deles não é atuante em iniciação científica e não participa de projetos de pesquisa.Essa participação é importante e se faz necessária, pois traz um ganho no desenvolvimento pessoal e insere o aluno em um ambiente multidisciplinar, que será importante para sua entrada no mercado de trabalho ${ }^{4}$.

Em um estudo realizado em seis escolas médicas, localizadas em diferentes estados, dos 413 alunos participantes, $28 \%$ não participavam de IC e $37 \%$ que participavam, era devido a uma disciplina optativa oferecida pelo instituição de ensino ${ }^{16}$.

Ao analisar, separadamente, por ciclos da formação médica, os alunos de ciclo básico são ainda menos participantes do que aqueles que se encontram no ciclo clínico, mas ainda assim, o número de atuantes não ultrapassou $50 \%$. Onde aqueles do ciclo clínico, estão em fase de apresentação de trabalho de conclusão de curso (TCC), o que pode influenciar sua entrada em grupos de pesquisa.

É importante que nesse processo, o aluno seja capaz de melhorar seu desempenho na escrita científica, objetivo que será alcançado mediante leitura crítica e frequente de demais trabalhos, de forma a proporcionar maior familiaridade com os termos específicos utilizados, além das normatizações metodológicas e de referências ${ }^{17}$.

A relevância do presente estudo está na evidência da deficiência do conhecimento em pesquisa científica, identificada pelas respostas dos alunos de medicina participantes. Porém os questionários foram aplicados anonimamente, impossibilitando a avaliação da veracidade dessas respostas, a identificação e a capacitação desse grupo.

\section{Conclusão}

A maioria dos estudantes de medicina não atua em iniciação científica e nunca participou de grupos de pesquisa, com evidência de grande desconhecimento a respeito das fases de execução de uma pesquisa, assim como do uso das plataformas essenciais para a atuação científica.

Há necessidade da realização de cursos e eventos que tenham como objetivo a disseminação do conhecimento científico, além da inserção precoce do aluno da graduação na pesquisa, incentivando, possibilitando e facilitando a sua inserção do estudante na iniciação científica. Assim, proporcionará que ele trabalhe gradualmente na construção do seu currículo e seja um multiplicador de conhecimento, mesmo após sua formação, unindo seu conhecimento clínico às atualizações científicas no tratamento de seus pacientes e na disseminação de informações aos demais membros de sua equipe de trabalho.

\section{Referências}

1. Fernandes PMP, Mariani AW. O ensino médico além da graduação: iniciação cienífica. Diagn Trat 2010;15(3):104-5.

2. Bernardi MM. A Importância da Iniciação Científica e Perspectivas de Atuação Profissional. Biológico 2003;65(1/2):101. 
3. Therrien SMN, Therrien J. Trabalhos científicos e o estado da questão. Estud em Avaliação Educ 2004;15(30):5-16.

4. Tenório MP, Beraldi G. Iniciação científica no Brasil e nos cursos de medicina. Rev Assoc Med Bras 2010; 56:390-3.

5. Ministério da Ciência Tecnologia e Inovações. Iniciação científica Portal CNPq [Internet]. [citado 5 de setembro de 2020]. Available at: http:// cnpq.br/iniciacao-cientifica

6. Cardoso GP, Silva Junior CT, Martinho J, Cyrillo RJT. Iniciação científica em medicina: uma questão de interesse para todas as especialidades. Pulmão RJ2004;13(1):8-12.

7. Araújo W. Desafios dos programas de iniciação científica no Brasil Notícias Concursos [Internet]. 2020 [citado 5 de setembro de 2020]. Available at: https://noticiasconcursos.com.br/educacao/desafios-dos-programas-deiniciacao-cientifica-no-brasil/

8. Bariani ICD. Estilos cognitivos de universitarios e iniciação cientifica. 1998. Tese de Doutorado. Repositório Digital da UNICAMP. 170p.

9. Puccini RF, Sampaio LO, Batista NA. A formação médica na Unifesp: excelência e compromisso social. Editora Fap-Unifesp; 2008. 312p.

10. Resende J, Alves R, Coutinho M, et al. Importância da Iniciação Científica e Projetos de Extensão para Graduação em Medicina. Rev Bras CiênSaúde 2013;17:11-8.

11. Calculadora Amostral - Comentto [Internet]. [citado 26 de março de 2021]. Available at: https://comentto.com/calculadora-amostral/

12. Ferreira DS, Oliveira JRM, Pitombeira SSR. A importância e os benefícios do currículo lattes. I Encontros Universitários da UFC. 2016:1

13. Estácio LSS. A importância do currículo lattes como ferramenta que representa a ciência, tecnologia e inovação no país. Rev ACB 2017;22(2):30011.

14. Loretto NRM. Plataforma Brasil: limites e desafios. Rev Cir Traumatol Buco-maxilo-fac2012; 12(1):8-12.

15. Cavalcante ASP, Vasconcelos MIO, Lira GV, et al. As Ligas Acadêmicas na Area da Saúde: Lacunas do Conhecimento na Produção Científica Brasileira. Rev Bras Educ Med 2018;42(1):199-206.

16. Oliveira NA, Alves LA, Luz MR. Iniciação científica na graduação: o que diz o estudante de medicina? Rev Bras Educ Med 2008;32(3):309-14.

17. Garcia FS. Metodologia da pesquisa científica: organização estrutural e os desafios para redigir o trabalho de conclusão [Internet]. [citado 26 de setembro de 2020]. Available at: http://www.uniesp.edu.br/fnsa/revista 